\title{
Unitarity sum rules, three site moose model, and the ATLAS 2 TeV diboson anomalies
}

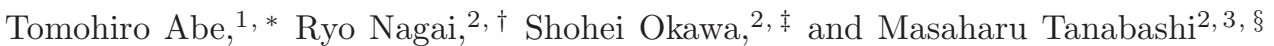 \\ ${ }^{1}$ Institute of Particle and Nuclear Studies, High Energy Accelerator Research Organization (KEK), Tsukuba 305-0801, Japan \\ 2 Department of Physics, Nagoya University, Nagoya 464-8602, Japan \\ ${ }^{3}$ Kobayashi-Maskawa Institute for the Origin of Particles and the Universe, Nagoya University, Nagoya 464-8602, Japan
}

(Dated: July 29, 2018)

\begin{abstract}
We investigate $W^{\prime}$ interpretations for the ATLAS $2 \mathrm{TeV}$ diboson anomalies. The roles of the unitarity sum rules, which ensure the perturbativity of the longitudinal vector boson scattering amplitudes, are emphasized. We find the unitarity sum rules and the custodial symmetry are powerful enough to predict various nontrivial relations among $W W Z^{\prime}, W Z W^{\prime}, W W h, W W^{\prime} h$ and $Z Z^{\prime} h$ coupling strengths in a model independent manner. We also perform surveys in the general parameter space of $W^{\prime}$ models and find the ATLAS $2 \mathrm{TeV}$ diboson anomalies may be interpreted as a $W^{\prime}$ particle of the three site moose model, i.e., a Kaluza-Klein like particle in a deconstructed extra dimension model. It is also shown that the non standard-model-like Higgs boson is favored by the present data to interpret the ATLAS diboson anomalies as the consequences of the $W^{\prime}$ and $Z^{\prime}$ bosons.

PACS numbers: 12.60.Cn,14.70.Pw,14.80.Rt
\end{abstract}

\section{INTRODUCTION}

Recently, the ATLAS Collaboration of the LHC experiment reported anomalies in their search for highmass diboson ( $W Z, W W$ or $Z Z$ ) resonances with bosontagged jets at the diboson invariant mass $2 \mathrm{TeV}[1]$. $W$ and $Z$ bosons resulting from high-mass resonance are highly boosted, so each boson's hadronic decay products are reconstructed as a single fat jet $J$ in this search. The reported local significances of anomalies are $3.4 \sigma$, $2.6 \sigma$, and $2.9 \sigma$ for $W Z \rightarrow J J, W W \rightarrow J J$, and $Z Z \rightarrow J J$ channels, respectively. If we explain the ATLAS $W Z \rightarrow J J$ anomaly in the $W^{\prime}$ model, we need to introduce a narrow high-mass $W^{\prime}$ boson with $M_{W^{\prime}}=2$ $\mathrm{TeV}, \Gamma_{W^{\prime}}<100 \mathrm{GeV}$, and

$$
\sigma\left(p p \rightarrow W^{\prime} ; \sqrt{s}=8 \mathrm{TeV}\right) B_{W^{\prime}}(W Z) \simeq 14 \mathrm{fb} .
$$

Note here the above cross section is the best fit value. A $W^{\prime}$ particle with a little bit smaller production cross section may also be consistent with the ATLAS diboson anomaly.

The CMS Collaboration also reported their results of search for high-mass diboson resonance in the same decay channel [2]. Although the CMS Collaboration reported a small excess around $1.8 \mathrm{TeV}$, at the resonance mass of $2 \mathrm{TeV}$, CMS only gives the upper limit on $\sigma B$, $\sigma\left(p p \rightarrow W^{\prime} ; \sqrt{s}=8 \mathrm{TeV}\right) B_{W^{\prime}}(W Z)<13 \mathrm{fb}$. The ATLAS Collaboration also reported their search for highmass diboson resonance $X$ in the $W V \rightarrow \ell \nu q \bar{q}$ decay channel [3]. Here $V$ stands for $W$ or $Z$. The ATLAS

\footnotetext{
*abetomo@post.kek.jp

†nagai@eken.phys.nagoya-u.ac.jp

† okawa@eken.phys.nagoya-u.ac.jp

$\S$ tanabash@eken.phys.nagoya-u.ac.jp
}

limit on $\sigma B$ in this decay channel is about

$$
\sum_{X} \sigma(p p \rightarrow X ; \sqrt{s}=8 \mathrm{TeV}) B_{X}(W V)<10 \mathrm{fb},
$$

for $2 \mathrm{TeV}$ narrow resonance $X$. The limit (I.2) causes a tension with the best fit value of the ATLAS diboson anomaly $\sigma\left(p p \rightarrow W^{\prime}\right) B_{W^{\prime}}(W Z) \simeq 14 \mathrm{fb}$. We use the value $\sigma B \simeq 10 \mathrm{fb}$ as a reference for our interpretation of the ATLAS diboson anomaly in this paper, though only $70 \%$ of the ATLAS diboson excess can be explained with this cross section.

The CMS Collaboration recently reported their search limit on the $W^{\prime}$ production in the $W^{\prime} \rightarrow W h \rightarrow J J$ decay channel in Ref. [4]. Although the CMS Collaboration found a small excess of event numbers around $M_{W^{\prime}}=1.8 \mathrm{TeV}$, they found rather severe upper limit for $M_{W^{\prime}}=2 \mathrm{TeV}$ resonance, i.e., about $\sigma\left(p p \rightarrow W^{\prime} ; \sqrt{s}=\right.$ $8 \mathrm{TeV}) B_{W^{\prime}}(W h)<7 \mathrm{fb}$. As we will see later, this upper limit causes a severer tension with the ATLAS diboson anomaly. In Ref. [5], the CMS Collaboration reported their search result for high-mass resonance $W^{\prime}$ in the decay channel $W^{\prime} \rightarrow W h \rightarrow \ell \nu b \bar{b}$. Again, they found an excess at $M_{W^{\prime}} \simeq 1.8 \mathrm{TeV}$.

After the ATLAS Collaboration reported the $2 \mathrm{TeV}$ diboson anomalies [1], many studies of possible theoretical interpretations have appeared in the market [6 17]. One of the biggest questions raised in these interpretations is whether the ATLAS diboson anomaly at $M=2$ $\mathrm{TeV}$ is related with the CMS excesses at $M=1.8 \mathrm{TeV}$ or not. Given the situation where the jet mass resolutions of ATLAS and CMS detectors are much better than 100 $\mathrm{GeV}$, it seems unlikely that the CMS $1.8 \mathrm{TeV}$ excesses are directly related with the ATLAS $2 \mathrm{TeV}$ anomalies, however. If this is not so, the next theoretical challenge is to make viable models of $W^{\prime}$ explaining the ATLAS diboson anomalies without causing conflicts with the CMS upper limit on the $W^{\prime} \rightarrow W h$ decay channel, $\sigma\left(p p \rightarrow W^{\prime}\right) B_{W^{\prime}}(W h)<7 \mathrm{fb}$ at $M_{W^{\prime}}=2 \mathrm{TeV}$. This 
is a tough challenge, however, if we take the CMS $7 \mathrm{fb}$ upper limit seriously. The Higgs boson is the $S U(2)_{W}$ partner of the would-be Nambu-Goldstone boson (NGB) in the Standard Model (SM). The equivalence theorem of the longitudinal $W$ boson and the eaten would-be NGB amplitudes then suggests us a relation

$$
\Gamma_{W^{\prime}}(W Z)=\Gamma_{W^{\prime}}(W h)
$$

for sufficiently heavy $W^{\prime}$. See, e.g, Ref. [18] for a typical $W^{\prime}$ model satisfying this relation (I.3). The relation (I.3) implies that the $W^{\prime}$ branching fraction to the $W h$ mode is identical to the $W^{\prime}$ branching fraction to $W Z$, i.e., $B_{W^{\prime}}(W Z)=B_{W^{\prime}}(W h)$. The CMS $7 \mathrm{fb}$ upper limit on the $W h$ channel therefore gives an upper limit on $\sigma(p p \rightarrow$ $\left.W^{\prime}\right) B(W Z)<7 \mathrm{fb}$. Less than only a half of the ATLAS diboson anomaly excess $\sim 14 \mathrm{fb}$ can be explained!

Although this tension may be explained by a statistical fluctuation at the present stage, it is tempting to consider scenarios free from the relation (I.3). In this paper, we point out that the relation (I.3) is true only if the couplings of the $125 \mathrm{GeV}$ Higgs boson with $W W$ and $Z Z$ are same as the SM predictions. We investigate the relation (I.3) from the viewpoint of the perturbative unitarity of the longitudinal $W$ and $W^{\prime}$ boson scattering amplitudes. We derive a set of unitarity sum rules among coupling strengths of $W^{\prime}$ and $W$ bosons, which should be satisfied in any perturbative model of $W^{\prime}$. We then obtain a relation among the $W Z W^{\prime}$ coupling, the $W W h$ coupling, and the $W h W^{\prime}$ coupling by using these unitarity sum rules. We find that, if the $125 \mathrm{GeV}$ Higgs is a non-SM Higgs boson, the relation (I.3) should be modified as

$$
\kappa_{V}^{2} \Gamma_{W^{\prime}}(W Z)=\Gamma_{W^{\prime}}(W h) .
$$

Here $\kappa_{V}$ is defined as $\kappa_{V}=g_{W W h} / g_{W W h}^{\mathrm{SM}}$, with $g_{W W h}$ and $g_{W W h}^{\mathrm{SM}}=g_{W} M_{W}$ being the $W W h$ coupling strength and its corresponding SM value, respectively. The ATLAS $2 \mathrm{TeV}$ diboson anomalies may be consistent with the CMS limits on the $W h$ decay channel, if we consider a model with $\kappa_{V}<1$. $^{1}$

Inspired by the unitarity sum rules and the custodial $S U(2)$ symmetry arguments, we then introduce a parametrization of $W^{\prime}$ and $Z^{\prime}$ couplings, and survey the parameter space to find phenomenologically viable models consistent with the existing limits on the $W^{\prime}$ and $Z^{\prime}$ particles. We then show that the three site moose model [19], a linear sigma model generalization of the three site Higgsless model [20], can explain the parameter space consistent both with the ATLAS anomalies and with the existing limits on $W^{\prime}$ and $Z^{\prime}$. Note that the three site Higgsless model is a deconstruction 21, 22. version of the extra dimension Higgsless model [23]. The

1 Here we assume existence of mechanism to adjust the Higgs measurement signal strengths with $\kappa_{V}<1$. gauge symmetry breaking structure of the three site moose model thus resembles the structure of extra dimension models containing bulk weak gauge fields. The $W^{\prime}$ and $Z^{\prime}$ bosons in the three site moose model can therefore be regarded as the Kaluza-Klein (KK) modes of the weak gauge bosons. We also note that, as emphasized in Ref. [19], the three site moose model implements a mechanism to adjust the Higgs signal strengths even with $\kappa_{V}<1$.

This paper is organized as follows: In Sec. III we derive a set of unitarity sum rules in a class of models with the custodial symmetry including arbitrary number of custodial $S U(2)$ triplet vector bosons $\left(W, W^{\prime}, W^{\prime \prime}, \cdots\right)$ and neutral Higgs bosons $\left(h_{1}, h_{2}, \cdots\right)$. We obtain a relationship between the $W W^{\prime} h$ coupling and the $W W h$ coupling by using the unitarity sum rules. We propose a parametrization for the $W^{\prime}$ and $Z^{\prime}$ couplings in a manner consistent with the perturbativity and the custodial symmetry in Sec. III] Surveys in the parameter space of $W^{\prime}$ and $Z^{\prime}$ models are presented in Sec. IV] Sec. V is devoted to the three site moose model. Summary and outlooks are presented in Sec. VI.

\section{UNITARITY SUM RULES}

In order to keep the perturbative unitarity in the longitudinal vector boson scattering amplitudes, in any perturbative model, self-interaction coupling strengths of massive vector bosons need to satisfy a set of unitarity sum rules 24 27. Examples of such unitarity sum rules for a model including a tower of massive vector bosons $\left(W, W^{\prime}, W^{\prime \prime}, \cdots\right)$ are presented in Ref. [28] in the context of the deconstructed Higgsless theory. Unitarity sum rules in a model with $W, W^{\prime}$ and neutral Higgs bosons $\left(h_{1}, h_{2}, \cdots\right)$ are discussed in Ref. [19]. See also Refs. [29 32]. In this section, we further generalize the unitarity sum rules of Ref. [19] to obtain a relationship between $W W h$ and $W W^{\prime} h$ couplings.

\section{A. General Sum Rules}

For simplicity, in this section, we consider the custodial $S U(2)$ symmetry limit. Effects of the custodial symmetry violation arising from the weak hypercharge gauge coupling will be discussed later. The model we consider contains $N_{V}$ custodial $S U(2)$ triplet vector bosons ( $W_{i \mu}^{a}$, $\left.a=1,2,3, i=0,1, \cdots, N_{V}-1\right)$ and $N_{h}$ singlet Higgs bosons $\left(h_{i}, i=1,2, \cdots, N_{h}\right)$. In order to cancel the $E^{4}$-behavior of the longitudinal $W_{i} W_{j} \rightarrow W_{k} W_{\ell}$ scattering amplitudes, quartic vector boson coupling strengths $g_{W_{i} W_{j} W_{k} W_{\ell}}$ need to satisfy

$$
g_{W_{i} W_{j} W_{k} W_{\ell}}=\sum_{m} g_{W_{i} W_{j} W_{m}} g_{W_{k} W_{\ell} W_{m}},
$$

with $g_{W_{i} W_{j} W_{k} W_{\ell}}$ being symmetric under the exchange of the indices $i, j, k, \ell$ (Bose symmetry). The triple vector 
boson couplings also satisfy the Bose symmetry. Here we specified the quartic and triple vector couplings by using notations similar to Ref. 19]. Using (II.1) and the Bose symmetry of $g_{W_{i} W_{j} W_{k} W_{\ell}}$, it is easy to see

$$
\begin{aligned}
\sum_{m} g_{W_{i} W_{j} W_{m}} g_{W_{k} W_{\ell} W_{m}} & =\sum_{m} g_{W_{i} W_{k} W_{m}} g_{W_{j} W_{\ell} W_{m}} \\
& =\sum_{m} g_{W_{i} W_{\ell} W_{m}} g_{W_{k} W_{j} W_{m}} .
\end{aligned}
$$

Requiring the cancellation of the $E^{2}$-behavior, we also obtain a sum rule which relates the Higgs coupling $g_{W_{i} W_{j} h_{k}}$ with the vector boson self-interaction couplings,

$$
\begin{aligned}
\sum_{m} g_{W_{i} W_{j} h_{m}} g_{W_{k} W_{\ell} h_{m}} & \left(M_{W_{i}}^{2}+M_{W_{j}}^{2}+M_{W_{k}}^{2}+M_{W_{\ell}}^{2}\right) g_{W_{i} W_{j} W_{k} W_{\ell}} \\
& -\sum_{m} M_{W_{m}}^{2} g_{W_{i} W_{j} W_{m}} g_{W_{k} W_{\ell} W_{m}} \\
& -\sum_{m} M_{W_{m}}^{2} g_{W_{i} W_{k} W_{m}} g_{W_{j} W_{\ell} W_{m}} \\
& -\sum_{m} M_{W_{m}}^{2} g_{W_{i} W_{\ell} W_{m}} g_{W_{k} W_{j} W_{m}} .
\end{aligned}
$$

Again we used notations similar to Ref. [19]. We also obtain

$$
\begin{gathered}
\sum_{m} \frac{\left(M_{W_{i}}^{2}-M_{W_{j}}^{2}\right)\left(M_{W_{k}}^{2}-M_{W_{\ell}}^{2}\right)}{M_{W_{m}}^{2}} g_{W_{i} W_{j} W_{m}} g_{W_{k} W_{\ell} W_{m}} \\
=\sum_{m} M_{W_{m}}^{2}\left(-g_{W_{i} W_{k} W_{m}} g_{W_{j} W_{\ell} W_{m}}\right. \\
\left.\quad+g_{W_{i} W_{\ell} W_{m}} g_{W_{k} W_{j} W_{m}}\right) .(\text { II. } 4)
\end{gathered}
$$

Note here that the RHS of (II.3) is symmetric under the exchange of the indices $i, j, k, \ell$. We therefore obtain

$$
\begin{aligned}
\sum_{m} g_{W_{i} W_{j} h_{m}} g_{W_{k} W_{\ell} h_{m}} & =\sum_{m} g_{W_{i} W_{k} h_{m}} g_{W_{j} W_{\ell} h_{m}} \\
& =\sum_{m} g_{W_{i} W_{\ell} h_{m}} g_{W_{k} W_{j} h_{m}} .
\end{aligned}
$$

\section{B. Properties of $W^{\prime}$}

We are now ready to discuss applications of the unitarity sum rules (I.1), (II.2), (I.3), (II.4) and (I.5).

We first consider the sum rule which ensures the cancellation of the $E^{4}$-behavior in the $W W \rightarrow W W$ amplitude. Eq. II.1 reads

$$
g_{W W W W}=g_{W W W}^{2}+g_{W W W^{\prime}}^{2} .
$$

We assume here that the sum rule is saturated only by $W\left(=W_{0}\right)$ and $W^{\prime}\left(=W_{1}\right)$. Effects of possibly existing heavier resonances $W^{\prime \prime}\left(=W_{2}\right), \cdots$, are assumed to be negligible.

We should note that the sum rule (II.6) is incomplete below the energy scale $E \lesssim M_{W^{\prime}}$, where the $W^{\prime}$ exchange term $g_{W W W^{\prime}}^{2}$ does not affect the $W W \rightarrow W W$ amplitude. The longitudinal polarization of $W$ gives a factor $E / M_{W}$ in the amplitude for $E \gg M_{W}$. For the energy scale $M_{W^{\prime}} \gtrsim E \gg M_{W}$, the $W W \rightarrow W W$ amplitude therefore behaves as

$$
\left(g_{W W W W}-g_{W W W}^{2}\right) \frac{E^{4}}{M_{W}^{4}} .
$$

Requiring the amplitude is still in a perturbative regime [33] at $E=M_{W^{\prime}}$, we obtain a condition

$$
\left(g_{W W W W}-g_{W W W}^{2}\right) \frac{M_{W^{\prime}}^{4}}{M_{W}^{4}} \lesssim 32 \pi .
$$

It is now easy to see that the $g_{W W W^{\prime}}$ coupling need to satisfy

$$
g_{W W W^{\prime}}^{2} \frac{M_{W^{\prime}}^{4}}{M_{W}^{4}} \lesssim 32 \pi .
$$

Parametrizing the $g_{W W W^{\prime}}$ coupling

$$
g_{W W W^{\prime}}=\xi_{V} g_{W W W} \frac{M_{W}^{2}}{M_{W^{\prime}}^{2}},
$$

the perturbativity condition (II.9) can be expressed as

$$
\left|\xi_{V}\right| \lesssim 15
$$

Here we used $g_{W W W} \simeq 0.65$. In typical analyses of collider phenomenologies of $W^{\prime}$, the parameter $\xi_{V}$ is chosen to be $\xi_{V} \simeq 1$. Although this choice clearly satisfies the perturbativity condition (II.11), it is also possible to construct models with larger value of $\xi_{V}$, e.g, $\xi_{V} \sim 5$, still keeping the perturbativity condition (II.9).

We next consider the sum rule which ensures the cancellation of the $E^{4}$-behavior in the $W W \rightarrow W^{\prime} W^{\prime}$ amplitude, Eq.(II.2),

$g_{W W^{\prime} W}^{2}+g_{W W^{\prime} W^{\prime}}^{2}=g_{W W W} g_{W^{\prime} W^{\prime} W}+g_{W W W^{\prime}} g_{W^{\prime} W^{\prime} W^{\prime}}$.

Eq. (II.12) can be regarded as a quadratic equation of $g_{W W^{\prime} W^{\prime}}$,

$$
\begin{aligned}
0= & g_{W W^{\prime} W^{\prime}}^{2}-g_{W W W} g_{W W^{\prime} W^{\prime}} \\
& +g_{W W W^{\prime}}^{2}-g_{W^{\prime} W^{\prime} W^{\prime}} g_{W W W^{\prime}} .
\end{aligned}
$$

Plugging (II.10) into (II.13), we obtain

$$
\begin{aligned}
0= & g_{W W^{\prime} W^{\prime}}^{2}-g_{W W W} g_{W W^{\prime} W^{\prime}} \\
& +\xi_{V}^{2} g_{W W W}^{2} \frac{M_{W}^{4}}{M_{W^{\prime}}^{4}} \\
& -\xi_{V} g_{W^{\prime} W^{\prime} W^{\prime}} g_{W W W} \frac{M_{W^{2}}^{2}}{M_{W^{\prime}}^{2}} .
\end{aligned}
$$


Solving the quadratic equation (II.14) in the $M_{W^{\prime}}^{2} \gg$ $M_{W}^{2}$ limit, we obtain two solutions

$$
g_{W W^{\prime} W^{\prime}}=g_{W W W},
$$

or

$$
g_{W W^{\prime} W^{\prime}}=-\xi_{V} g_{W^{\prime} W^{\prime} W^{\prime}} \frac{M_{W}^{2}}{M_{W^{\prime}}^{2}} .
$$

We next turn to the properties of Higgs couplings $g_{W W h_{m}}$ and $g_{W W^{\prime} h_{m}}$. Let us start with the $E^{2}$ sum rule for the $W W \rightarrow W W$ amplitude. Using (II.3) we obtain

$$
\begin{aligned}
\sum_{m} g_{W W h_{m}}^{2}= & 4 M_{W}^{2}\left(g_{W W W}^{2}+g_{W W W^{\prime}}^{2}\right) \\
& -3 M_{W}^{2} g_{W W W}^{2}-3 M_{W^{\prime}}^{2} g_{W W W^{\prime}}^{2}
\end{aligned}
$$

where III.1 is also used to rewrite the quartic vector boson coupling strength $g_{W W W W}$ in terms of $g_{W W W}$ and $g_{W W W^{\prime}}$. Plugging (II.10) into (II.17), we find

$$
\sum_{m} g_{W W h_{m}}^{2}=M_{W}^{2} g_{W W W}^{2}\left[1-3 \xi_{V}^{2} \frac{M_{W}^{2}}{M_{W^{\prime}}^{2}}\right]
$$

The Higgs boson coupling with the $W W$ state is therefore affected by the parameter $\xi_{V}$ and the $W^{\prime}$ boson mass. The roles of the unitarity sum rule (II.18) have been widely studied in Refs. [29 32].

The $E^{2}$ sum rules for the $W W \rightarrow W W^{\prime}$ amplitude can also be derived from (II.3),

$$
\begin{aligned}
& \sum_{m} g_{W W h_{m}} g_{W W^{\prime} h_{m}} \\
&=\left(3 M_{W}^{2}+M_{W^{\prime}}^{2}\right)\left[g_{W W W} g_{W W^{\prime} W}+g_{W W W^{\prime}} g_{W W^{\prime} W^{\prime}}\right] \\
&-3 M_{W}^{2} g_{W W W} g_{W W^{\prime} W}-3 M_{W^{\prime}}^{2} g_{W W W^{\prime}} g_{W W^{\prime} W^{\prime}} \\
&= M_{W^{\prime}}^{2} g_{W W W} g_{W W W^{\prime}} \\
&+\left(3 M_{W}^{2}-2 M_{W^{\prime}}^{2}\right) g_{W W W^{\prime}} g_{W W^{\prime} W^{\prime}} .
\end{aligned}
$$

Putting the parametrization of $g_{W W W^{\prime}}$ (II.10) and one solution of $g_{W W^{\prime} W^{\prime}}$ (II.15) into (II.19), we obtain

$$
\sum_{m} g_{W W h_{m}} g_{W W^{\prime} h_{m}}=-\xi_{V} M_{W}^{2} g_{W W W}^{2}\left[1+\mathcal{O}\left(\frac{M_{W}^{2}}{M_{W^{\prime}}^{2}}\right)\right]
$$

If we put (II.10) and the other solution of $g_{W W^{\prime} W^{\prime}}$ (II.16), we find

$$
\sum_{m} g_{W W h_{m}} g_{W W^{\prime} h_{m}}=\xi_{V} M_{W}^{2} g_{W W W}^{2}\left[1+\mathcal{O}\left(\frac{M_{W}^{2}}{M_{W^{\prime}}^{2}}\right)\right]
$$

For the $W W \rightarrow W^{\prime} W^{\prime}$ scattering, not only (II.3) but also (II.4) gives non-trivial sum rules. From (II.3), we obtain

$$
\begin{aligned}
\sum_{m} & g_{W W^{\prime} h_{m}}^{2} \\
= & \left(2 M_{W}^{2}+2 M_{W^{\prime}}^{2}\right)\left[g_{W W^{\prime} W}^{2}+g_{W W^{\prime} W^{\prime}}^{2}\right] \\
& -2\left[M_{W}^{2} g_{W W^{\prime} W}^{2}+M_{W^{\prime}}^{2} g_{W W^{\prime} W}^{2}\right] \\
& -\left[M_{W}^{2} g_{W W W^{\prime}} g_{W^{\prime} W^{\prime} W}+M_{W^{\prime}}^{2} g_{W W W^{\prime}} g_{W^{\prime} W^{\prime} W^{\prime}}\right] \\
= & 2 M_{W^{\prime}}^{2} g_{W W W^{\prime}}^{2}+2 M_{W^{\prime}}^{2} g_{W W^{\prime} W^{\prime}}^{2} \\
& -\left[M_{W}^{2} g_{W W W} g_{W W^{\prime} W^{\prime}}+M_{W^{\prime}}^{2} g_{W W W^{\prime}} g_{W^{\prime} W^{\prime} W^{\prime}}\right]
\end{aligned}
$$

We also obtain from (II.4

$$
\begin{aligned}
& \frac{\left(M_{W}^{2}-M_{W^{\prime}}^{2}\right)^{2}}{M_{W}^{2}} g_{W W^{\prime} W}^{2}+\frac{\left(M_{W}^{2}-M_{W^{\prime}}^{2}\right)^{2}}{M_{W^{\prime}}^{2}} g_{W W^{\prime} W^{\prime}}^{2} \\
& =M_{W}^{2}\left(g_{W W^{\prime} W}^{2}-g_{W W W} g_{W^{\prime} W^{\prime} W}\right) \\
& \quad+M_{W^{\prime}}^{2}\left(g_{W W^{\prime} W^{\prime}}^{2}-g_{W W W^{\prime}} g_{W^{\prime} W^{\prime} W^{\prime}}\right), \text { (II.23) }
\end{aligned}
$$

which reads

$$
\begin{aligned}
& M_{W}^{2} g_{W W W} g_{W W^{\prime} W^{\prime}}+M_{W^{\prime}}^{2} g_{W W W^{\prime}} g_{W^{\prime} W^{\prime} W^{\prime}} \\
& =\left[M_{W}^{2}-\frac{\left(M_{W}^{2}-M_{W^{\prime}}^{2}\right)^{2}}{M_{W}^{2}}\right] g_{W W W^{\prime}}^{2} \\
& \quad+\left[M_{W^{\prime}}^{2}-\frac{\left(M_{W}^{2}-M_{W^{\prime}}^{2}\right)^{2}}{M_{W^{\prime}}^{2}}\right] g_{W W^{\prime} W^{\prime}}^{2} .
\end{aligned}
$$

Note that the last line of (II.22) can be erased by using (II.24). We now have

$$
\sum_{m} g_{W W^{\prime} h_{m}}^{2}=\frac{M_{W^{\prime}}^{4}}{M_{W}^{2}} g_{W W W^{\prime}}^{2}+\frac{M_{W}^{4}}{M_{W^{\prime}}^{2}} g_{W W^{\prime} W^{\prime}}^{2} .
$$

It is now easy to show

$$
\sum_{m} g_{W W^{\prime} h_{m}}^{2}=\xi_{V}^{2} M_{W}^{2} g_{W W W}^{2}\left[1+\mathcal{O}\left(\frac{M_{W}^{2}}{M_{W^{\prime}}^{2}}\right)\right] .
$$

Combining (II.18), (II.20) and (II.26), we obtain an impressive sum rule

$$
\frac{1}{M_{W}^{2}} \sum_{m}\left(g_{W W^{\prime} h_{m}}+\xi_{V} g_{W W h_{m}}\right)^{2}=\xi_{V}^{2} g_{W W W}^{2} \mathcal{O}\left(\frac{M_{W}^{2}}{M_{W^{\prime}}^{2}}\right) .
$$

Similarly, using (II.21) instead of (II.20), we find

$$
\frac{1}{M_{W}^{2}} \sum_{m}\left(g_{W W^{\prime} h_{m}}-\xi_{V} g_{W W h_{m}}\right)^{2}=\xi_{V}^{2} g_{W W W}^{2} \mathcal{O}\left(\frac{M_{W}^{2}}{M_{W^{\prime}}^{2}}\right) .
$$

For both cases, we obtain

$$
g_{W W^{\prime} h_{m}}= \pm \xi_{V}\left[g_{W W h_{m}} \pm g_{W W h}^{\mathrm{SM}} \mathcal{O}\left(\frac{M_{W}}{M_{W^{\prime}}}\right)\right] .
$$

We used here $g_{W W h}^{\mathrm{SM}} \simeq g_{W W W} M_{W}$. The Higgs $(h)$ coupling with $W W^{\prime}$ is therefore related with $g_{W W W^{\prime}}$ and $g_{W W h}$ through the relation (II.29) in the large $M_{W^{\prime}}$ 
limit. If the coupling of the $125 \mathrm{GeV}$ Higgs boson $(h)$ $g_{W W h} \simeq g_{W W h}^{\mathrm{SM}}$, we find the uncertainty in (II.29) is about $4 \%\left(=M_{W} / M_{W^{\prime}}\right)$ for $M_{W^{\prime}}=2 \mathrm{TeV}$ and therefore is negligibly small.

We note (II.29) is a novel relation, which has not been pointed out in earlier references. The relation provides us further information for the $W^{\prime}$ boson properties beyond the widely studied $W W \rightarrow W W$ sum rule (II.18). We emphasize that we provided the proof of (II.29) in models with arbitrary number of the neutral Higgs bosons. Another remark is that the conclusion (II.29) is unchanged even if we consider models containing $W^{\prime \prime}$ or higher KK resonances. The result (II.29) can therefore be applied to a large class of perturbative models which include the $W^{\prime}$ particle and neutral Higgs bosons.

\section{UNITARITY INSPIRED PARAMETRIZATION FOR $W^{\prime}$ AND $Z^{\prime}$}

In the previous section, we have shown that the coupling strengths of the $W^{\prime}$ boson can be controlled by the perturbative unitarity requirements in the custodial $S U(2)$ symmetric model. Especially, we found

$$
g_{W W W^{\prime}}=\xi_{V} g_{W W W} \frac{M_{W}^{2}}{M_{W^{\prime}}^{2}}, \quad \xi_{V} \lesssim 15,
$$

and

$$
g_{W W^{\prime} h_{m}}= \pm \xi_{V} g_{W W h_{m}} .
$$

In this section, we consider effects of custodial $S U(2)$ symmetry violation $M_{Z} \neq M_{W}, M_{Z^{\prime}} \neq M_{W^{\prime}}$, requiring the high energy $E^{2}$-behavior of the longitudinal $W$ and $Z$ boson scattering amplitudes are custodial $S U(2)$ symmetric. This requirement is justified because the weak hypercharge coupling (the origin of the custodial symmetry violation) does not affect the $E^{2}$-behavior in the amplitudes at the tree level. We find (III.1) needs to be modified $\mathrm{as}^{2}$

$$
\begin{gathered}
g_{W Z W^{\prime}}=\xi_{V} g_{W} \frac{M_{W} M_{Z}}{M_{W^{\prime}}^{2}} . \\
g_{W W Z^{\prime}}=\xi_{V} g_{W} \frac{M_{W}^{2}}{M_{Z^{\prime}}^{2}} R,
\end{gathered}
$$

with $R\left(M_{W^{\prime}} / M_{Z^{\prime}}\right)$ being a function of $M_{W^{\prime}} / M_{Z^{\prime}}$ satisfying $R(1)=1$. Here $g_{W}$ stands for the gauge coupling strength of the $W$ boson.

The Higgs couplings with the $W W^{\prime}$ and $Z Z^{\prime}$ states can also be parametrized by using the custodial $S U(2)$ symmetry. We obtain

$$
g_{W W^{\prime} h}=\xi_{h} g_{W} M_{W},
$$

\footnotetext{
2 The default value of the PYTHIA [34] implementation of the extended gauge model [35] corresponds to $\xi_{V}=M_{W}^{2} / M_{Z}^{2}$.
}

$$
g_{Z Z^{\prime} h}=\xi_{h} g_{W} M_{Z} R,
$$

with $h$ being the $125 \mathrm{GeV}$ Higgs boson. The requirement of the perturbative unitarity then leads to

$$
\xi_{h}= \pm \kappa_{V} \xi_{V}, \quad \kappa_{V} \equiv \frac{g_{W W h}}{g_{W} M_{W}}
$$

as we have shown in the previous section. It is now straightforward to show the relation (I.4). The relation (III.7) and therefore (I.4) should be regarded as one of the most important unitarity relations obtained in this paper. We are now able to describe the physics of $W$, $W^{\prime}$ and Higgs by using the two parameters $\left(\kappa_{V}\right.$ and $\left.\xi_{V}\right)$, instead of the three $\left(\kappa_{V}, \xi_{V}\right.$ and $\left.\xi_{h}\right)$.

In order to study collider phenomenologies of $W^{\prime}$ and $Z^{\prime}$ particles, we need to specify the couplings of $W^{\prime}$ and $Z^{\prime}$ with quarks and leptons. In this paper we adopt an ansatz in which $W^{\prime}$ and $Z^{\prime}$ couple with weak current of quarks (leptons) with universal coupling strength $\xi_{q} g_{W}$ $\left(\xi_{\ell} g_{W}\right)$. An example of model satisfying this ansatz will be introduced in Sec. V

\section{FIT TO THE ATLAS DIBOSON ANOMALY}

We are now ready to search the parameter region of $\xi_{V}, \xi_{q}$, and $\xi_{\ell}$ so as to explain the ATLAS $2 \mathrm{TeV}$ diboson anomaly in $W^{\prime}$ models. As we discussed in Sec. [. we use

$$
\sum_{X} \sigma(p p \rightarrow X) B_{X}(V V) \simeq 10 \mathrm{fb},
$$

as a reference value to explain the ATLAS $2 \mathrm{TeV}$ diboson anomaly. Here $X$ is a narrow width new particle having $2 \mathrm{TeV}$ mass decaying to the $V V$ states, with $V$ being a weak gauge boson $W$ or $Z$. The ATLAS Collaboration reported excesses not only in the $W Z$ category, but also in the $W W$ and $Z Z$ categories. It has being claimed that there exist significant size of event contamination among $W Z, W W$ and $Z Z$ categories in the $J J$ events [6, 14], however. We therefore evaluate

$$
\sum_{X=W^{\prime}, Z^{\prime}} \sigma(p p \rightarrow X) B_{X}(V V)
$$

for a degenerated $W^{\prime}$ and $Z^{\prime}$ model $\left(M_{W^{\prime}}=M_{Z^{\prime}}=2\right.$ $\mathrm{TeV})$, and

$$
\sigma\left(p p \rightarrow W^{\prime}\right) B_{W^{\prime}}(W Z)
$$

for a non-degenerated model $\left(M_{Z^{\prime}}>M_{W^{\prime}}=2 \mathrm{TeV}\right)$, and simply compare the number with the reference value (IV.1).

In addition to $\sigma B$, we also need to explain the narrow width of the new particle $X$, typically smaller than the bin size of the experiment[1], $100 \mathrm{GeV}$.

The model explaining the diboson anomaly need to be consistent with the existing limits on the $W^{\prime}$ and $Z^{\prime}$ bosons. Both ATLAS and CMS experiments report upper limits on the production cross section of $W^{\prime}$ in its 
leptonic decay channels [36, 37]. For $2 \mathrm{TeV} W^{\prime}$ boson search in $p p$ collisions at $\sqrt{s}=8 \mathrm{TeV}$, the limit is

$$
\sigma\left(p p \rightarrow W^{\prime}\right) B_{W^{\prime}}(\ell \nu) \lesssim 0.4 \mathrm{fb} .
$$

Limits on the $Z^{\prime} \rightarrow e^{+} e^{-}, \mu^{+} \mu^{-}$are reported in Refs. 38, 39]. For $M_{Z^{\prime}}=2 \mathrm{TeV}$, these references give a limit

$$
\sigma\left(p p \rightarrow Z^{\prime}\right) B_{Z^{\prime}}\left(\ell^{+} \ell^{-}\right) \lesssim 0.2 \mathrm{fb} .
$$

The LHC limits on the resonant dijet production can also be used to constrain $W^{\prime}$ models. Using the limits presented in Refs. [40, 41], we see

$$
\sum_{X=W^{\prime}, Z^{\prime}} \sigma(p p \rightarrow X) B_{X}(2 j) \lesssim 100 \mathrm{fb},
$$

for a degenerated $M_{W^{\prime}}=M_{Z^{\prime}}=2 \mathrm{TeV}$ model, and

$$
\sigma\left(p p \rightarrow W^{\prime}\right) B_{W^{\prime}}(2 j) \lesssim 100 \mathrm{fb},
$$

for a non-degenerated model with $M_{W^{\prime}}=2 \mathrm{TeV}$.

Finally, the model needs to satisfy the limit on the $W^{\prime} \rightarrow W h$ and $Z^{\prime} \rightarrow Z h$ decay modes. Here $h$ stands for the $125 \mathrm{GeV}$ Higgs particle. The limit quoted in Ref.[4] is

$$
\sum_{X=W^{\prime}, Z^{\prime}} \sigma(p p \rightarrow X) B_{X}(V h) \lesssim 7 \mathrm{fb}
$$

for $M_{W^{\prime}}=M_{Z^{\prime}}=2 \mathrm{TeV}$, and

$$
\sigma\left(p p \rightarrow W^{\prime}\right) B_{W^{\prime}}(W h) \lesssim 7 \mathrm{fb},
$$

for a non-degenerated model with $M_{W^{\prime}}=2 \mathrm{TeV}$.

Figure 1 shows these limits in the $\xi_{f}-\xi_{V}$ plane for $M_{Z^{\prime}}=M_{W^{\prime}}=2 \mathrm{TeV}$. We assume quark-lepton universal coupling $\xi_{f}=\left|\xi_{q}\right|=\left|\xi_{\ell}\right|$ in this plot. We also assume the $125 \mathrm{GeV}$ Higgs coupling with $W W^{\prime}$ and $Z Z^{\prime}$ are given by

$$
\xi_{h}= \pm \xi_{V}
$$

which corresponds to $\kappa_{V}=1$ in the unitarity relation (III.7). The dijet limit (IV.6) is applied for the resonant production cross section of five flavor $q \bar{q}$ pairs. The $W^{\prime}$ and $Z^{\prime}$ particles are produced through their couplings with quarks in $p p$ collisions at $8 \mathrm{TeV}$. The production cross sections are evaluated by using the CTEQ6L1 set of the parton distribution functions [42].

We see in this plot that the Higgs mode limit (IV.8) and the leptonic decay mode limit (IV.4) rule out huge parameter space. It is impossible to obtain the reference value of the cross section (IV.1) without causing conflicts with the Higgs mode limit (IV.8) under the Higgs coupling assumption (IV.10). We are only able to achieve $\sum_{X=W^{\prime}, Z^{\prime}} \sigma(X) B_{X}(V V) \simeq 7 \mathrm{fb}$ at most.

A similar plot for a leptophobic $\xi_{\ell}=0$ model is shown in Figure 2 assuming the degenerated $W^{\prime}$ and $Z^{\prime}$, $M_{Z^{\prime}}=M_{W^{\prime}}=2 \mathrm{TeV}$. Again, the $125 \mathrm{GeV}$ Higgs coupling is assumed to satisfy (IV.10). Although the constraints from the leptonic decay channels of $W^{\prime}$ and $Z^{\prime}$

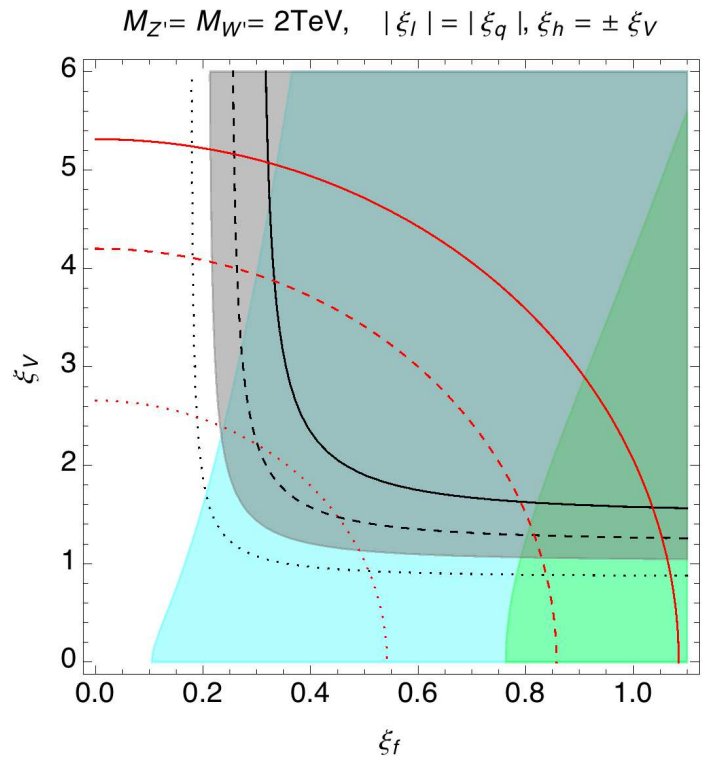

FIG. 1: Limits on the $W^{\prime}$ and $Z^{\prime}$ couplings in the $\xi_{f}-\xi_{V}$ plane for the degenerated $M_{Z^{\prime}}=M_{W^{\prime}}=2 \mathrm{TeV}$ model. $\xi_{f}=$ $\left|\xi_{q}\right|=\left|\xi_{\ell}\right|$ and $\xi_{h}= \pm \xi_{V}$ are assumed. The darkgreen region, the lightblue region, and the gray region are excluded by the dijet mode limit (IV.6), the $\ell \nu$ mode (IV.4), and Higgs mode (IV.8), respectively. Although we do not show the limit from (IV.5) in the plot, it is numerically almost identical to the $W^{\prime} \rightarrow \ell \nu$ limit. The black solid curve, the black dashed curve, and the black dotted curve are for $\sigma\left(p p \rightarrow W^{\prime}\right) B_{W^{\prime}}(W Z)+$ $\sigma\left(p p \rightarrow Z^{\prime}\right) B_{Z^{\prime}}(W W)=15 \mathrm{fb}, 10 \mathrm{fb}$, and $5 \mathrm{fb}$, respectively. The red solid curve, the red dashed curve, and the red dotted curve are for $\Gamma_{W^{\prime}}=80 \mathrm{GeV}, 50 \mathrm{GeV}$, and $20 \mathrm{GeV}$, respectively. The width of $Z^{\prime}$ is almost equal to $\Gamma_{W^{\prime}}$ thanks to the custodial symmetry.

disappear in the leptophobic model, the limit on the $V h$ channel gives severe constraint on (IV.2). It is impossible to obtain the the reference value $10 \mathrm{fb}$ in this setup.

In order to explain the diboson excess without causing conflicts with the Higgs mode limit (IV.8), we need to take smaller value of $\xi_{h}$. The unitarity relation (III.7) suggests us such a value of $\xi_{h}$ can be achieved only if we consider models with a non-SM like Higgs $\left(\kappa_{V}<1\right)$. Figure 3 shows the plot with $\xi_{h} / \xi_{V}= \pm 0.7$, i.e., $\kappa_{V}=$ 0.7. The quark-lepton universal couplings $\xi_{f}=\left|\xi_{q}\right|=$ $\left|\xi_{\ell}\right|$ are assumed in the plot. The reference cross section value for the excess can be explained at, e.g., $\xi_{V} \simeq 4$ and $\xi_{f} \simeq 0.23$. Note that the choice of this parameter $\xi_{V}$ satisfies the perturbativity condition (II.11).

We next consider the non-degenerated case, $M_{Z^{\prime}}>$ $M_{W^{\prime}}=2 \mathrm{TeV}$. The $Z^{\prime}$ boson is assumed to be heavy enough to be separated from the $2 \mathrm{TeV}$ resonance. The plot corresponding to this model is shown in Figure 4 Here $\xi_{f}=\left|\xi_{\ell}\right|=\left|\xi_{q}\right|$ and $\xi_{h} / \xi_{V}= \pm 0.7$ are assumed. We find that the reference cross section value for the ATLAS diboson anomaly can be explained at, e.g., $\xi_{V} \simeq 4$ and $\xi_{f} \simeq 0.28$.

A plot similar to Figures 1 2, and 3 is also presented in Ref. [6] in the context of the techni- $\rho$ interpretation 


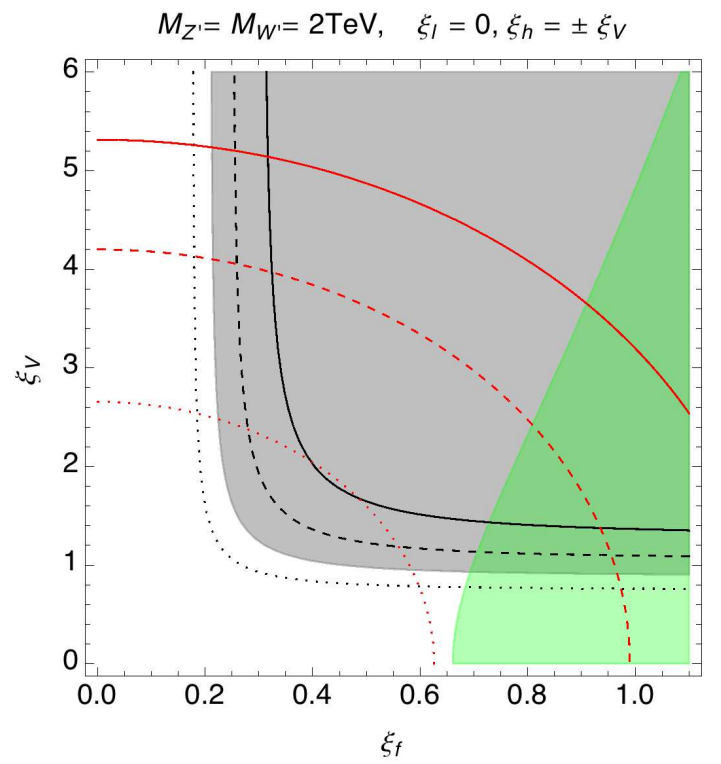

FIG. 2: Plot similar to Figure 1 for leptophobic case $\xi_{\ell}=0$, $\xi_{f}=\left|\xi_{q}\right| . \xi_{h}= \pm \xi_{V}$ is assumed. The darkgreen region, and the gray region are excluded by the dijet mode limit (IV.6), and Higgs mode (IV.8), respectively. The black solid curve, the black dashed curve, and the black dotted curve are for $\sigma\left(p p \rightarrow W^{\prime}\right) B_{W^{\prime}}(W Z)+\sigma\left(p p \rightarrow Z^{\prime}\right) B_{Z^{\prime}}(W W)=15 \mathrm{fb}, 10$ $\mathrm{fb}$, and $5 \mathrm{fb}$, respectively. The red solid curve, the red dashed curve, and the red dotted curve are for $\Gamma_{W^{\prime}}=80 \mathrm{GeV}, 50 \mathrm{GeV}$, and $20 \mathrm{GeV}$, respectively. The width of $Z^{\prime}$ is almost equal to $\Gamma_{W^{\prime}}$ thanks to the custodial symmetry.

for the ATLAS diboson anomalies. The plot presented in the latest version of Ref. [6] seems to be consistent with our results.

\section{THREE SITE MOOSE MODEL}

So far, we have analyzed the unitarity sum rules and the interpretations of the LHC anomaly of the diboson resonance in terms of $W^{\prime}$ and $Z^{\prime}$ without writing an explicit gauge invariant Lagrangian.

In this section, we introduce the three site moose model [20] as an example to explain the ATLAS diboson excess in a perturbative manner. We are able to check explicitly that the unitarity sum rules are satisfied in the three site moose model. We also find that the parameter region explaining the ATLAS diboson anomaly is naturally realized in this model.

The three site moose model 20] was originally introduced as a deconstruction version of the Higgsless theory 23]. This model contains $W^{\prime}$ and $Z^{\prime}$ bosons as KK particles of electroweak gauge bosons. These KK particles are, at least partly, responsible for the unitarization of the longitudinal weak gauge boson scattering amplitudes 23, 43, 44]. After the LHC discovery of the $125 \mathrm{GeV}$ Higgs boson, the three site moose model was extended to include the $125 \mathrm{GeV}$ Higgs particle in Ref. [19].

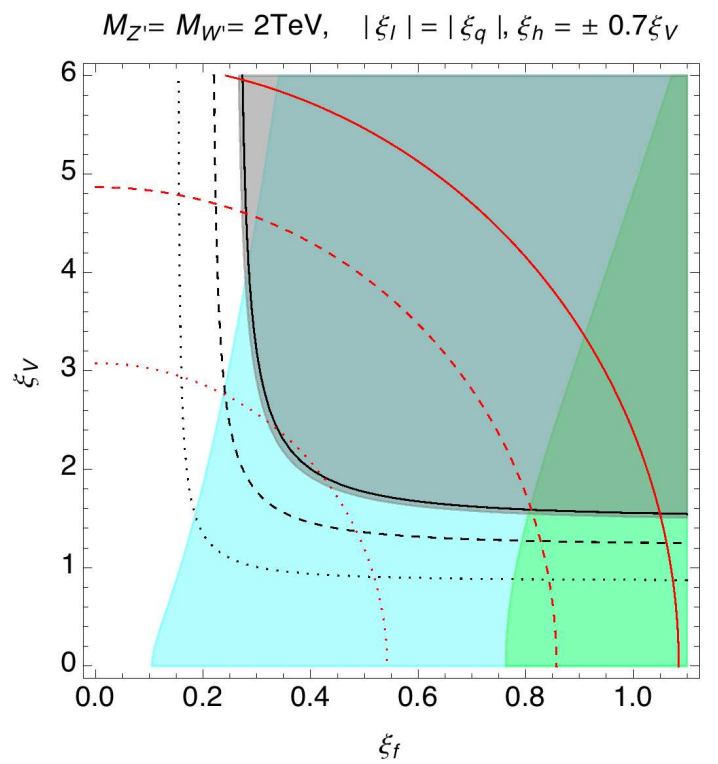

FIG. 3: Plot similar to Figure 1 for $\xi_{h} / \xi_{V}= \pm 0.7 . \quad \xi_{f}=$ $\left|\xi_{q}\right|=\left|\xi_{\ell}\right|$ is assumed. The darkgreen region, the lightblue region, and the gray region are excluded by the dijet mode limit (IV.6), the $\ell \nu$ mode (IV.4), and Higgs mode (IV.8), respectively. Although we do not show the limit from (IV.5) in the plot, it is numerically almost identical to the $W^{\prime} \rightarrow \ell \nu$ limit. The black solid curve, the black dashed curve, and the black dotted curve are for $\sigma\left(p p \rightarrow W^{\prime}\right) B_{W^{\prime}}(W Z)+\sigma(p p \rightarrow$ $\left.Z^{\prime}\right) B_{Z^{\prime}}(W W)=15 \mathrm{fb}, 10 \mathrm{fb}$, and $5 \mathrm{fb}$, respectively. The red solid curve, the red dashed curve, and the red dotted curve are for $\Gamma_{W^{\prime}}=80 \mathrm{GeV}, 50 \mathrm{GeV}$, and $20 \mathrm{GeV}$, respectively. The width of $Z^{\prime}$ is almost equal to $\Gamma_{W^{\prime}}$ thanks to the custodial symmetry.

This model can be regarded as a benchmark model to study the phenomenologies of $W^{\prime}$ and $Z^{\prime}$ particles.

The structure of gauge symmetry breaking in the three site moose model is illustrated in the "moose notation" 45] in Figure [5. In the three site moose model, we introduce $S U(2)_{W 0} \times S U(2)_{W 1} \times U(1)_{Y 2}$ gauge groups. The line connecting the $S U(2)_{W 0}$ group with the $S U(2)_{W 1}$ group in the moose diagram represents bifundamental $(2 \times 2$ matrix $)$ Higgs field $\Phi_{1}$,

$$
\Phi_{1}=S_{1} \mathbf{1}+i \tau^{a} \pi_{1}^{a},
$$

with $\tau^{a}$ being the Pauli spin matrix. Similarly, the line between the $S U(2)_{W 1}$ and $U(1)_{Y 2}$ gauge groups corresponds to the Higgs field $\Phi_{2}$

$$
\Phi_{2}=S_{2} \mathbf{1}+i \tau^{a} \pi_{2}^{a} .
$$

The covariant derivatives of $\Phi_{1}$ and $\Phi_{2}$ are given by

$$
\begin{gathered}
D_{\mu} \Phi_{1}=\partial_{\mu} \Phi_{1}+i g_{W 0} W_{0 \mu} \Phi_{1}-i g_{W 1} \Phi_{1} W_{1 \mu}, \\
D_{\mu} \Phi_{2}=\partial_{\mu} \Phi_{2}+i g_{W 1} W_{1 \mu} \Phi_{2}-i g_{Y 2} \Phi_{2} \frac{\tau^{3}}{2} B_{\mu} .
\end{gathered}
$$

Here $W_{0 \mu}=W_{0 \mu}^{a} \frac{\tau^{a}}{2}, W_{1 \mu}=W_{1 \mu}^{a} \frac{\tau^{a}}{2}$, and $B_{\mu}$ are the gauge fields of $S U(2)_{W 0}, S U(2)_{W 1}$ and $U(1)_{Y 2}$. 


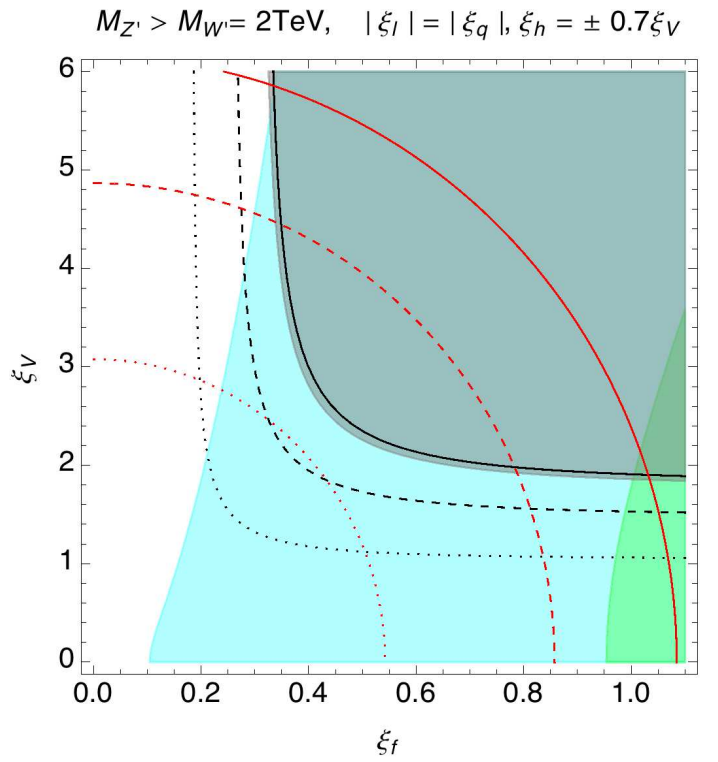

FIG. 4: Plot similar to Figure 1 for non-degenerated model $M_{Z^{\prime}}>M_{W^{\prime}}=2 \mathrm{TeV}$ with $\xi_{h} / \xi_{V}= \pm 0.7 . \quad \xi_{f}=\left|\xi_{q}\right|=\left|\xi_{\ell}\right|$ is assumed. The darkgreen region, the lightblue region, and the gray region are excluded by the dijet mode limit (IV.6), the $\ell \nu$ mode (IV.4), and Higgs mode (IV.9), respectively. The black solid curve, the black dashed curve, and the black dotted curve are for $\sigma\left(p p \rightarrow W^{\prime}\right) B_{W^{\prime}}(W Z)=15 \mathrm{fb}, 10 \mathrm{fb}$, and $5 \mathrm{fb}$, respectively. The red solid curve, the red dashed curve, and the red dotted curve are for $\Gamma_{W^{\prime}}=80 \mathrm{GeV}, 50 \mathrm{GeV}$, and 20 $\mathrm{GeV}$, respectively.

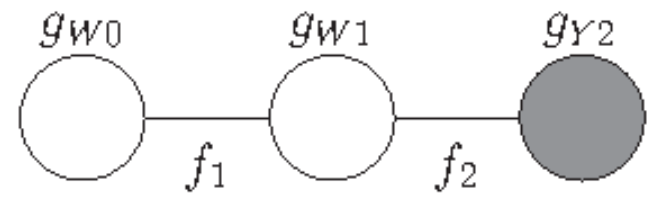

FIG. 5: The moose diagram for the three site model. The blank circles represent $S U(2)$ gauge groups, with coupling strengths $g_{W 0}$ and $g_{W 1}$, and the shaded circle is a $U(1)$ gauge group with coupling $g_{Y 2}$.

The Higgs field $\Phi_{1}$ is assumed to acquire its vacuum expectation value (VEV),

$$
\left\langle\Phi_{1}\right\rangle=f_{1} \mathbf{1}
$$

which breaks the $S U(2)_{W_{0}} \times S U(2)_{W 1}$ into the diagonal subgroup $S U(2)$. Similarly, the $S U(2)_{W 1} \times U(1)_{Y 2}$ is broken to $U(1)$ thanks to the $\mathrm{VEV}$ of $\Phi_{2}$,

$$
\left\langle\Phi_{2}\right\rangle=f_{2} \mathbf{1}
$$

Simultaneous existence of two VEVs $f_{1} \neq 0$ and $f_{2} \neq 0$ therefore leads to the spontaneous symmetry breaking pattern

$$
S U(2)_{W 0} \times S U(2)_{W 1} \times U(1)_{Y 2} \rightarrow U(1)_{\mathrm{em}} .
$$

Diagonalizing the mass matrices of $W_{0 \mu}, W_{1 \mu}$ and $B_{\mu}$ which arise from the Higgs kinetic term Lagrangian

$$
\mathcal{L} \ni \frac{1}{4} \operatorname{tr}\left[\left(D_{\mu} \Phi_{1}\right)^{\dagger}\left(D^{\mu} \Phi_{1}\right)\right]+\frac{1}{4} \operatorname{tr}\left[\left(D_{\mu} \Phi_{2}\right)^{\dagger}\left(D^{\mu} \Phi_{2}\right)\right]
$$

we obtain mass eigenstates, $W^{\prime}, Z^{\prime}$ (heavier massive charged and neutral vector bosons), $W, Z$ (lighter massive vector bosons), and a massless photon.

The weak hypercharge gauge couplings of quarks and leptons are given by

$$
\mathcal{L} \ni-J_{Y, \text { quark }}^{\mu} B_{\mu}-J_{Y, \text { lepton }}^{\mu} B_{\mu} .
$$

As shown in Ref. [20], after the gauge symmetry breaking, the weak currents of quarks and leptons can be "delocalized" with delocalization parameters $x_{q}$ and $x_{\ell}$ $\left(0 \leq x_{q} \leq 1,0 \leq x_{\ell} \leq 1\right)$,

$$
\begin{aligned}
\mathcal{L} \ni & -J_{W, \text { quark }}^{a \mu}\left(g_{W 0} W_{0 \mu}^{a}\left(1-x_{q}\right)+g_{W 1} W_{1 \mu}^{a} x_{q}\right) \\
& -J_{W, \text { lepton }}^{a \mu}\left(g_{W 0} W_{0 \mu}^{a}\left(1-x_{\ell}\right)+g_{W 1} W_{1 \mu}^{a} x_{\ell}\right) .
\end{aligned}
$$

We should emphasize here that the weak current of quarks and leptons couples with both $S U(2)_{W 0}$ and $S U(2)_{W 1}$ in Eq. (V.10). This phenomenon called "delocalization" is characteristic in extra dimension scenarios 46, in which KK quarks and KK leptons exist. Similar phenomenon also realizes in the partial compositeness scenarios 47] in which the role of the KK fermions is played by the composite fermions. This is in contrast to the conventional G221 models (including right-handed $S U(2)$ model), in which the weak current cannot be delocalized.

The delocalization parameters $x_{q}$ and $x_{\ell}$ need to be flavor universal in order to avoid the flavor-changingneutral-current constraints in the three site model [48]. The quark delocalization parameter $x_{q}$ can differ from the lepton parameter $x_{\ell}$, however. The electroweak precision constraints can be satisfied for heavy enough $W^{\prime}$ and $Z^{\prime}$. Even with lighter $M_{W^{\prime}} \lesssim 1 \mathrm{TeV}$, we are able to suppress the Peskin-Takeuchi $S-T$ parameters [49, 50] if we choose the lepton delocalization parameter $x_{\ell}$ to the value determined by the "ideal delocalization" [51]. As we will see later, the delocalization parameters $x_{q}$ and $x_{\ell}$ are related with the $W^{\prime}$ couplings with quarks and leptons $\xi_{q}$ and $\xi_{\ell}$. Assuming the quark lepton universality of the $W^{\prime}$ coupling $\xi_{q}=\xi_{\ell}$, we are thus able to express the electroweak precision observable parameters in terms of $\xi_{f}=\left|\xi_{q}\right|=\left|\xi_{\ell}\right|$. In this section, we will also check whether or not the region in the $\xi_{f}-\xi_{V}$ plane favored by the ATLAS diboson anomalies is consistent with the electroweak precision measurements.

We have two neutral Higgs bosons in this model. One degree of freedom $\left(h_{1}\right)$ of neural Higgs arises from $\Phi_{1}$, the other $\left(h_{2}\right)$ from $\Phi_{2}$. The charged and pseudoscalar components of $\Phi_{1}$ and $\Phi_{2}$, i.e., $\pi_{1}^{a}$ and $\pi_{2}^{a}$ in (V.1 and (V.2) are all eaten by massive gauge bosons $W, W^{\prime}, Z$ 
and $Z^{\prime}$. The $125 \mathrm{GeV}$ Higgs boson $h$ is considered to be a mixture of $h_{1}$ and $h_{2}$,

$$
h=h_{1} \cos \alpha+h_{2} \sin \alpha,
$$

where

$$
S_{1}=f_{1}+h_{1}, \quad S_{2}=f_{2}+h_{2} .
$$

We are now ready to discuss the ATLAS $2 \mathrm{TeV}$ diboson anomaly in the three site moose model. There are three possible ways to obtain the hierarchy $M_{Z^{\prime}}, M_{W^{\prime}}=$ $2 \mathrm{TeV} \gg M_{Z}, M_{W}$ in this setup. One option is to take $g_{W 1} \gg g_{W 0}, g_{Y 2}$ with keeping the VEVs $f_{1}=f_{2}$ at the weak scale. Collider phenomenologies in this option were studied $^{3}$ in detail in Ref. [58 60]. This limit is theoretically interesting, because it can be regarded as an effective theory of strongly interacting Higgs sector 61 63. motivated by models of hidden local symmetry [64 68].

However, in order to realize $2 \mathrm{TeV} M_{W^{\prime}}$ with this option, we need non-perturbatively strong $g_{W 1}$. Ref. [6] studies an interpretation of the ATLAS diboson anomaly with $g_{W 1} \gg g_{W 0}, g_{Y 2}$, introducing higher order operators to suppress the effective coupling of the heavy vector resonance. We do not pursue this direction in this paper.

Other options are to take $f_{1} \gg f_{2}$ or $f_{1} \ll f_{2}$, keeping perturbative coupling constants $g_{W 0}, g_{W 1}$ and $g_{Y 2}$. In the subsections below, we will give our results of $M_{W^{\prime}}$, $M_{Z^{\prime}}, g_{W W Z^{\prime}}, g_{W Z W^{\prime}}, g_{W W h}$ and $g_{W W^{\prime} h}$ in these limits and check the unitarity sum rules explicitly in this model. We will also point out that the reciprocality between $\xi_{f}$ and $\xi_{V}$, suggested by the favored parameter regions of the ATLAS diboson anomaly fit, $\xi_{V} \simeq 3 \sim 5, \xi_{f} \simeq 0.2 \sim 0.3$ can be naturally realized in this setup.

$$
\text { A. } f_{1} \gg f_{2}
$$

We start with the case $f_{1} \gg f_{2}$. In this case the $S U(2)_{W 0} \times S U(2)_{W 1}$ gauge symmetry is broken into the diagonal subgroup at the high energy scale $f_{1}$, while the weak scale is given by $f_{2}$. We thus obtain the masses of $W^{\prime}$ and $Z^{\prime}$ in proportional to $f_{1}$,

$$
M_{W^{\prime}}^{2} \simeq M_{Z^{\prime}}^{2} \simeq \frac{g_{W 0}^{2}+g_{W 1}^{2}}{4} f_{1}^{2} .
$$

The weak $S U(2)$ gauge group at the weak scale should be the diagonal subgroup of $S U(2)_{W 0} \times S U(2)_{W 1}$, while the weak scale $U(1)_{Y}$ is given by $U(1)_{Y 2}$. The gauge

\footnotetext{
3 Hadron collider phenomenologies of narrow spin-1 resonances in the technicolor models are studied in Refs. [52, [53]. The $f_{1}=f_{2}$ model is believed to be a low energy effective description of the technicolor models. See also Refs. 54 57 for collider phenomenologies of techni-vector mesons in the low scale technicolor model.
}

coupling strengths at the weak scale are therefore

$$
g_{W}^{2} \simeq \frac{g_{W 0}^{2} g_{W 1}^{2}}{g_{W 0}^{2}+g_{W 1}^{2}}, \quad g_{Y}^{2} \simeq g_{Y 2}^{2} .
$$

The masses of the $W$ and $Z$ bosons are given by

$$
M_{W}^{2} \simeq \frac{g_{W}^{2}}{4} f_{2}^{2}, \quad M_{Z}^{2} \simeq \frac{g_{W}^{2}+g_{Y}^{2}}{4} f_{2}^{2} .
$$

It is easy to check these formulas by an explicit diagonalization of the mass matrices of the gauge fields $W_{0}$, $W_{1}$ and $B$, which are given by the Higgs kinetic term Lagrangian (V.8). We also obtain

$$
M_{Z^{\prime}}^{2}-M_{W^{\prime}}^{2}=\left(M_{Z}^{2}-M_{W}^{2}\right) \mathcal{O}\left(\frac{M_{W}^{2}}{M_{W^{\prime}}^{2}}\right) .
$$

The $W^{\prime}$ and $Z^{\prime}$ bosons are therefore highly degenerated in this setup. We next consider the $W Z W^{\prime}$ and $W W Z^{\prime}$ couplings. Explicit calculation of the mass diagonalization matrices of neutral and charged gauge bosons leads to

$$
g_{W Z W^{\prime}}=\frac{g_{W 1}}{g_{W 0}} g_{W} \frac{M_{W} M_{Z}}{M_{W^{\prime}}^{2}},
$$

and

$$
g_{W W Z^{\prime}}=\frac{g_{W 1}}{g_{W 0}} g_{W} \frac{M_{W}^{2}}{M_{Z^{\prime}}^{2}} .
$$

These results are perfectly consistent with our parametrization formulas (III.3) and (III.4). Therefore these couplings satisfy the unitarity and the custodial symmetry. Comparing (V.17), (V.18) with (III.3) and (III.4), we find $\xi_{V}$ in this model is given by

$$
\xi_{V}=\frac{g_{W 1}}{g_{W 0}} .
$$

We also obtain $R=1$, consistent with the custodial symmetry $M_{W^{\prime}}=M_{Z^{\prime}}$.

It is straightforward to calculate the Higgs couplings. We obtain

$$
\begin{gathered}
g_{W W h} \simeq g_{W} M_{W} \sin \alpha, \\
g_{W W^{\prime} h} \simeq-\frac{g_{W 1}}{g_{W 0}} g_{W} M_{W} \sin \alpha, \\
g_{Z Z^{\prime} h} \simeq-\frac{g_{W 1}}{g_{W 0}} g_{W} M_{Z} \sin \alpha .
\end{gathered}
$$

Again, these results are consistent with our custodial symmetry formulas (III.5) and (III.6) and the result of the unitarity sum rules (III.7). The parameter $\xi_{h}$ and $\kappa_{V}$ in this model are given by

$$
\xi_{h}=-\frac{g_{W 1}}{g_{W 0}} \sin \alpha,
$$




$$
\kappa_{V}=\sin \alpha .
$$

We next calculate the $W^{\prime}$ and $Z^{\prime}$ couplings with the quarks and the leptons. We find that both the quark hypercharge current and the lepton hypercharge current couple with the $Z^{\prime}$ boson only with coefficients suppressed by $\left(M_{Z}^{2}-M_{W}^{2}\right) / M_{W^{\prime}}^{2}$. The couplings of $W^{\prime}$ and $Z^{\prime}$ with the quarks and the leptons are therefore consistent with the ansatz given in Section. III The parameters $\xi_{q}$ and $\xi_{\ell}$ are given by

$$
\begin{aligned}
& \xi_{q}=\frac{g_{W 0}}{g_{W 1}}\left(1-x_{q}-x_{q} \frac{g_{W 1}^{2}}{g_{W 0}^{2}}\right), \\
& \xi_{\ell}=\frac{g_{W 0}}{g_{W 1}}\left(1-x_{\ell}-x_{\ell} \frac{g_{W 1}^{2}}{g_{W 0}^{2}}\right),
\end{aligned}
$$

with $x_{q}, x_{\ell}$ being the delocalization parameters for the quarks and the leptons. We note that, if the delocalization parameters are small enough $x_{q} \lesssim g_{W 0}^{2} / g_{W 1}^{2}, \xi_{V}$ and $\xi_{q}\left(\xi_{\ell}\right)$ satisfy the relation

$$
\xi_{V} \xi_{q} \lesssim 1
$$

Note that this relation is consistent with our result presented in Section. IV] The three site model with $g_{W 0} / g_{W 1} \simeq 0.25$ gives the reference value cross section for the ATLAS diboson anomalies without causing conflicts with other limits on $W^{\prime}$ and $Z^{\prime}$. The $\kappa_{V}$ is smaller than unity in this model, however. We need to take care the consistency with the signal strengths in the Higgs production measurements as done in Ref. [19].

The electroweak precision observable parameters are evaluated at the tree level as

$$
\hat{S} \simeq \frac{M_{W}^{2}}{M_{W^{\prime}}^{2}} \xi_{V} \xi_{f}, \quad W \simeq \frac{M_{W}^{2}}{M_{W^{\prime}}^{2}} \xi_{f}^{2} .
$$

Here we used the notation of Ref. [69]. We assumed $\xi_{f}=$ $\left|\xi_{q}\right|=\left|\xi_{\ell}\right|$ and neglected terms suppressed by $1 / \xi_{V}$ or $\xi_{f}$ in (V.28), given the situation that $\xi_{V} \simeq 3 \sim 4$ and $\xi_{f} \simeq 0.2 \sim 0.3$ are favored in the fit for the ATLAS diboson anomalies. The analysis of Ref. [69] shows that $\hat{S}$ and $W$ need to be smaller than a few permil in order to satisfy the electroweak precision constraints. Note that both $\hat{S}$ and $W$ are suppressed by $M_{W}^{2} / M_{W^{\prime}}^{2} \simeq 1.6 \times$ $10^{-3}$ in (V.28) and vanish in the ideal delocalization limit $\xi_{f}=0$. Thanks to the suppression factor $M_{W}^{2} / M_{W^{\prime}}^{2}$, the explanation of the ATLAS diboson anomaly is marginally consistent with the electroweak precision measurements at the tree level.

There also exist order of a few permil loop corrections to the electroweak precision parameters. However, these loop corrections depends on the assumptions of the UV completion behind the fermion delocalization. For an example, the fermion delocalization can be UV-completed by introducing additional heavy fermions. The loop level corrections to the electroweak precision parameters depends on the mass spectrum of the heavy additional fermions. See, e.g., Ref. [70]. Since we do not specify such a UV-completion in this paper, we do not consider loop level constraints any further.

$$
\text { B. } f_{1} \ll f_{2}
$$

The case $f_{1} \ll f_{2}$ can be studied in a similar manner. In this case the $S U(2)_{W 1} \times U(1)_{Y 2}$ group is broken to $U(1)_{Y}$ at the high energy scale $f_{2}$, while the $S U(2)_{W 0} \times$ $U(1)_{Y}$ is broken by $f_{1}$ at the weak scale. The masses of $W^{\prime}$ and $Z^{\prime}$ are therefore given by

$$
\begin{gathered}
M_{W^{\prime}}^{2} \simeq \frac{1}{4} g_{W 1}^{2} f_{2}^{2}, \\
M_{Z^{\prime}}^{2} \simeq \frac{1}{4}\left(g_{W 1}^{2}+g_{Y 2}^{2}\right) f_{2}^{2} .
\end{gathered}
$$

The $Z^{\prime}$ boson is heavier and can be separated from $W^{\prime}$ at the LHC experiments. The model in this limit therefore corresponds to the non-degenerated case in Section. IV of this paper. Since the weak hypercharge gauge boson at the weak scale is a mixture of the $S U(2)_{W 1}$ and $U(1)_{Y 2}$ gauge bosons, the gauge coupling strengths at the weak scale are given by

$$
g_{W}^{2} \simeq g_{W 0}^{2}, \quad g_{Y}^{2} \simeq \frac{g_{W 1}^{2} g_{Y 2}^{2}}{g_{W 1}^{2}+g_{Y 2}^{2}},
$$

and we obtain the weak gauge boson mass,

$$
M_{W}^{2} \simeq \frac{g_{W}^{2}}{4} f_{1}^{2}, \quad M_{Z}^{2} \simeq \frac{g_{W}^{2}+g_{Y}^{2}}{4} f_{1}^{2} .
$$

The $W Z W^{\prime}, W W Z^{\prime}, W W h, W W^{\prime} h, Z Z^{\prime} h$ couplings are given by

$$
\begin{gathered}
g_{W Z W^{\prime}}=\frac{g_{W 1}}{g_{W 0}} g_{W} \frac{M_{W} M_{Z}}{M_{W^{\prime}}^{2}}, \\
g_{W W Z^{\prime}}=\frac{g_{W 1}}{g_{W 0}} g_{W} \frac{M_{W}^{2}}{M_{Z^{\prime}}^{2}} \frac{M_{W^{\prime}}}{M_{Z^{\prime}}}, \\
g_{W W h} \simeq g_{W} M_{W} \cos \alpha, \\
g_{W W^{\prime} h} \simeq \frac{g_{W 1}}{g_{W 0}} g_{W} M_{W} \cos \alpha, \\
g_{Z Z^{\prime} h} \simeq \frac{g_{W 1}}{g_{W 0}} g_{W} M_{Z} \frac{M_{W^{\prime}}}{M_{Z^{\prime}}} \cos \alpha,
\end{gathered}
$$

which are consistent with our unitarity and custodial symmetry formulas (III.3), (III.4), (III.5), (III.6), and (III.7). The parameters $\xi_{V}, \xi_{h}, \kappa_{V}$, and $R$ are given by

$$
\begin{gathered}
\xi_{V}=\frac{g_{W 1}}{g_{W 0}} \\
\xi_{h}=\frac{g_{W 1}}{g_{W 0}} \cos \alpha
\end{gathered}
$$




$$
\kappa_{V}=\cos \alpha
$$

and

$$
R=\frac{M_{W^{\prime}}}{M_{Z^{\prime}}}
$$

The $Z^{\prime}$ boson does couple with the fermion hypercharge currents in this setup. Therefore the couplings of $Z^{\prime}$ with quarks and leptons cannot be parametrized by the parameters $\xi_{q}$ and $\xi_{\ell}$. The $Z^{\prime}$ boson becomes heavier enough than $M_{W^{\prime}}=2 \mathrm{TeV}$, however, and therefore irrelevant in the explanation of the ATLAS diboson anomalies. In the phenomenological analysis, we therefore use the parameters which describe the $W^{\prime}$ coupling with quarks and leptons,

$$
\begin{gathered}
\xi_{q}=-\frac{g_{W 1}}{g_{W 0}} x_{q}, \\
\xi_{\ell}=-\frac{g_{W 1}}{g_{W 0}} x_{\ell} .
\end{gathered}
$$

We find that the relatively small value of $\left|\xi_{q}\right| \simeq 0.3$, which is favored by the $W^{\prime}$ constraints, is possible if we take $x_{q} \lesssim g_{W 0}^{2} / g_{W 1}^{2}$.

We find a non-decoupling tree level correction

$$
\hat{S} \simeq-\frac{\xi_{f}}{\xi_{V}},
$$

which is not suppressed by $M_{W}^{2} / M_{W^{\prime}}^{2}$ in the $f_{1} \ll f_{2}$ model in contrast to the $f_{1} \gg f_{2}$ case. The constraint from the electroweak precision parameters is therefore much severer than the $f_{1} \gg f_{2}$ case in this setup. Again we assumed $\xi_{f}=\left|\xi_{q}\right|=\left|\xi_{\ell}\right|$ and neglected terms suppressed by $1 / \xi_{V}$ or $\xi_{f}$. For $\xi_{V} \simeq 3 \sim 5$ and $\xi_{f} \simeq$ $0.2 \sim 0.3$, we find $\hat{S} \simeq 0.1$, clearly contradicting with the present experimental limit on $\hat{S}$, namely $|\hat{S}| \lesssim 10^{-3}[69]$. The three site moose model with $f_{1} \ll f_{2}$ is therefore ruled out as an interpretation of the ATLAS diboson anomaly at least for the quark-lepton universal coupling case $\xi_{q}=\xi_{\ell}$.

\section{SUMMARY}

In this paper, we have studied general structures of perturbative $W^{\prime}$ models from the viewpoint of unitarity sum rules and the custodial $S U(2)$ symmetry. We found that the unitarity sum rules and the custodial symmetry are powerful enough, to predict many relations among $W W Z^{\prime}, W Z W^{\prime}, W W h, W W^{\prime} h$ and $Z Z^{\prime} h$ coupling strengths. Especially, we derived a novel relation (II.29) from these sum rules, which can be applied to a large class of perturbative models including arbitrary numbers of the heavy vector triplet bosons and neutral Higgs bosons. Using these relations, we surveyed parameter space of $W^{\prime}$ models to search for the region possible to explain the ATLAS $2 \mathrm{TeV}$ diboson anomalies. If the CMS excesses at $1.8 \mathrm{TeV}$ are not related with the ATLAS $2 \mathrm{TeV}$ anomalies, and if the $125 \mathrm{GeV}$ Higgs boson is the SM-like Higgs boson, we found that the CMS upper limit on the $W^{\prime} \rightarrow W h$ channel at $2 \mathrm{TeV}$ is hardly compatible with the ATLAS $2 \mathrm{TeV}$ diboson anomalies, suggesting non SM-like properties of the $125 \mathrm{GeV}$ Higgs boson. Based on the three site moose model Lagrangian, we then provided a couple of example models of non-SM like Higgs bosons, which may be able to explain the ATLAS diboson anomalies.

We emphasize that the three site moose model we used in this paper should be regarded merely as an example to illustrate the properties of models which may explain the ATLAS $2 \mathrm{TeV}$ diboson anomalies. Models having similar properties (the non SM-like Higgs and the delocalization of the fermion weak current) such as the extra dimension models, the partial compositeness models [47], the top triangle moose models [71, 72], and the composite Higgs models 73 76] should be studied further. Note that the proof of the relation (II.29) we provided in this paper is directly applicable only in a category of perturbative models with the custodial symmetry containing arbitrary numbers of heavy vector triplet and neutral Higgs bosons. Although this category of models already covers wide varieties of interesting models, there also exist phenomenologically viable models which do not belong to this category. It is particularly interesting to study the $W^{\prime}$ phenomenology in these non-perturbative models.

Each of ATLAS and CMS experiments will accumulate $\sim 10 \mathrm{fb}^{-1}$ luminosity within year 2015 run at $\sqrt{s}=13$ $\mathrm{TeV}$. If the ATLAS diboson anomalies are settled to exist in these LHC Run2 experiments, the next target is to clarify the properties of the $2 \mathrm{TeV}$ diboson resonance. Especially, as we stressed in this paper, its $V h$ decay channel $(V=W$ or $Z$ ) becomes important, since it can determine whether the $125 \mathrm{GeV}$ Higgs particle is SM-like or not.

\section{Acknowledgements}

We thank Junji Hisano, Sekhar R. Chivukula, Hidenori Fukano, Masafumi Kurachi, Shinya Matsuzaki and Koichi Yamawaki for useful discussions and valuable comments. T.A.'s work is supported by Grant-in-Aid for Scientific research from the Ministry of Education, Culture, Sports, Science and Technology (MEXT), Japan, No. 23104006. R.N.'s work is supported by Research Fellowships of the Japan Society for the Promotion of Science (JSPS) for Young Scientists No.263947. M.T.'s work is supported in part by the JSPS Grant-in-Aid for Scientific Research 15K05047. 


\section{Note added}

After we sent our first version of manuscript to the arXiv, a study on the unitarity implications for the AT-
LAS diboson anomalies had appeared [17], in which the $W W \rightarrow W W$ sum rule (II.18) was used to constrain the $W W h$ coupling.
[1] G. Aad et al. [ATLAS Collaboration], "Search for high-mass diboson resonances with boson-tagged jets in proton-proton collisions at $\sqrt{s}=8 \mathrm{TeV}$ with the ATLAS detector," arXiv:1506.00962 [hep-ex].

[2] V. Khachatryan et al. [CMS Collaboration], "Search for massive resonances in dijet systems containing jets tagged as $\mathrm{W}$ or $\mathrm{Z}$ boson decays in pp collisions at $\sqrt{s}=8$ TeV," JHEP 1408 (2014) 173 arXiv:1405.1994 [hep-ex]].

[3] G. Aad et al. [ATLAS Collaboration], "Search for production of $W W / W Z$ resonances decaying to a lepton, neutrino and jets in $p p$ collisions at $\sqrt{s}=8 \mathrm{TeV}$ with the ATLAS detector," arXiv:1503.04677 [hep-ex].

[4] V. Khachatryan et al. [CMS Collaboration], "Search for a massive resonance decaying into a Higgs boson and a $\mathrm{W}$ or $\mathrm{Z}$ boson in hadronic final states in proton-proton collisions at sqrt(s) =8 TeV," arXiv:1506.01443 [hep-ex].

[5] CMS Collaboration, "Search for massive $W h$ resonances decaying to the $\ell \nu b \bar{b}$ final state in the boosted regime at $\sqrt{s}=8 \mathrm{TeV}, "$ CMS PAS EXO-14-010.

[6] H. S. Fukano, M. Kurachi, S. Matsuzaki, K. Terashi and K. Yamawaki, "2 TeV Walking Technirho at LHC?," arXiv:1506.03751 [hep-ph].

[7] J. Hisano, N. Nagata and Y. Omura, "Interpretations of the ATLAS Diboson Resonances," arXiv:1506.03931 [hep-ph].

[8] D. B. Franzosi, M. T. Frandsen and F. Sannino, "Diboson Signals via Fermi Scale Spin-One States," arXiv:1506.04392 [hep-ph].

[9] K. Cheung, W. Y. Keung, P. Y. Tseng and T. C. Yuan, "Interpretations of the ATLAS Diboson Anomaly," arXiv:1506.06064 [hep-ph].

[10] B. A. Dobrescu and Z. Liu, "A $W^{\prime}$ boson near $2 \mathrm{TeV}$ : predictions for Run 2 of the LHC," arXiv:1506.06736 [hep$\mathrm{ph}]$.

[11] J. A. Aguilar-Saavedra, "Triboson interpretations of the ATLAS diboson excess," arXiv:1506.06739 [hep-ph].

[12] A. Alves, A. Berlin, S. Profumo and F. S. Queiroz, "Dirac-Fermionic Dark Matter in $U(1)_{X}$ Models," arXiv:1506.06767 [hep-ph].

[13] Y. Gao, T. Ghosh, K. Sinha and J. H. Yu, "G221 Interpretations of the Diboson and Wh Excesses," arXiv:1506.07511 [hep-ph].

[14] A. Thamm, R. Torre and A. Wulzer, "A composite Heavy Vector Triplet in the ATLAS di-boson excess," arXiv:1506.08688 [hep-ph].

[15] J. Brehmer, J. Hewett, J. Kopp, T. Rizzo and J. Tattersall, "Symmetry Restored in Dibosons at the LHC?," arXiv:1507.00013 [hep-ph].

[16] Q. H. Cao, B. Yan and D. M. Zhang, "Simple NonAbelian Extensions and Diboson Excesses at the LHC," arXiv:1507.00268 [hep-ph].

[17] G. Cacciapaglia and M. T. Frandsen, "Unitarity implications of diboson resonance in the $\mathrm{TeV}$ region for Higgs physics," arXiv:1507.00900 [hep-ph].

[18] D. Pappadopulo, A. Thamm, R. Torre and A. Wulzer,
"Heavy Vector Triplets: Bridging Theory and Data," JHEP 1409 (2014) 060 arXiv:1402.4431 [hep-ph]].

[19] T. Abe, N. Chen and H. J. He, "LHC Higgs Signatures from Extended Electroweak Gauge Symmetry," JHEP 1301 (2013) 082 arXiv:1207.4103 [hep-ph]].

[20] R. S. Chivukula, B. Coleppa, S. Di Chiara, E. H. Simmons, H. J. He, M. Kurachi and M. Tanabashi, "A Three Site Higgsless Model," Phys. Rev. D 74 (2006) 075011 hep-ph/0607124.

[21] N. Arkani-Hamed, A. G. Cohen and H. Georgi, "(De)constructing dimensions," Phys. Rev. Lett. 86 (2001) 4757 hep-th/0104005.

[22] C. T. Hill, S. Pokorski and J. Wang, "Gauge invariant effective Lagrangian for Kaluza-Klein modes," Phys. Rev. D 64 (2001) 105005 hep-th/0104035.

[23] C. Csaki, C. Grojean, H. Murayama, L. Pilo and J. Terning, "Gauge theories on an interval: Unitarity without a Higgs," Phys. Rev. D 69 (2004) 055006 hep-ph/0305237.

[24] J. M. Cornwall, D. N. Levin and G. Tiktopoulos, "Uniqueness of spontaneously broken gauge theories," Phys. Rev. Lett. 30 (1973) 1268 [Erratum-ibid. 31 (1973) 572].

[25] J. M. Cornwall, D. N. Levin and G. Tiktopoulos, "Derivation of Gauge Invariance from High-Energy Unitarity Bounds on the s M atrix," Phys. Rev. D 10 (1974) 1145 [Erratum-ibid. D 11 (1975) 972].

[26] C. H. Llewellyn Smith, "High-Energy Behavior and Gauge Symmetry," Phys. Lett. B 46 (1973) 233.

[27] J. F. Gunion, H. E. Haber and J. Wudka, "Sum rules for Higgs bosons," Phys. Rev. D 43 (1991) 904.

[28] R. S. Chivukula, H. J. He, M. Kurachi, E. H. Simmons and M. Tanabashi, "General Sum Rules for WW Scattering in Higgsless Models: Equivalence Theorem and Deconstruction Identities," Phys. Rev. D 78 (2008) 095003 arXiv:0808.1682 [hep-ph]].

[29] R. Foadi, M. Jarvinen and F. Sannino, "Unitarity in Technicolor," Phys. Rev. D 79 (2009) 035010 arXiv:0811.3719 [hep-ph]].

[30] A. E. Carcamo Hernandez and R. Torre, "A 'Composite' scalar-vector system at the LHC," Nucl. Phys. B 841 (2010) 188 arXiv:1005.3809 [hep-ph]].

[31] B. Bellazzini, C. Csaki, J. Hubisz, J. Serra and J. Terning, "Composite Higgs Sketch," JHEP 1211 (2012) 003 arXiv:1205.4032 [hep-ph]].

[32] C. Englert, P. Harris, M. Spannowsky and M. Takeuchi, "Unitarity-controlled resonances after Higgs discovery," arXiv:1503.07459 [hep-ph].

[33] B. W. Lee, C. Quigg and H. B. Thacker, "Weak Interactions at Very High-Energies: The Role of the Higgs Boson Mass,' ' Phys. Rev. D 16 (1977) 1519.

[34] T. Sjostrand, S. Mrenna and P. Z. Skands, "A Brief Introduction to PYTHIA 8.1," Comput. Phys. Commun. 178 (2008) 852 arXiv:0710.3820 [hep-ph]].

[35] G. Altarelli, B. Mele and M. Ruiz-Altaba, "Searching for 
New Heavy Vector Bosons in $p \bar{p}$ Colliders," Z. Phys. C 45 (1989) 109 [Z. Phys. C 47 (1990) 676].

[36] G. Aad et al. [ATLAS Collaboration], "Search for new particles in events with one lepton and missing transverse momentum in $p p$ collisions at $\sqrt{s}=8 \mathrm{TeV}$ with the ATLAS detector," JHEP 1409 (2014) 037 arXiv:1407.7494 [hep-ex]].

[37] V. Khachatryan et al. [CMS Collaboration], "Search for physics beyond the standard model in final states with a lepton and missing transverse energy in proton-proton collisions at sqrt(s) =8 TeV," Phys. Rev. D 91 (2015) 9, 092005 arXiv:1408.2745 [hep-ex]].

[38] G. Aad et al. [ATLAS Collaboration], "Search for highmass dilepton resonances in pp collisions at $\sqrt{s}=8 \mathrm{TeV}$ with the ATLAS detector," Phys. Rev. D 90 (2014) 5, 052005 arXiv:1405.4123 [hep-ex]].

[39] V. Khachatryan et al. [CMS Collaboration], "Search for physics beyond the standard model in dilepton mass spectra in proton-proton collisions at $\sqrt{s}=8 \mathrm{TeV}$," JHEP 1504 (2015) 025 arXiv:1412.6302 [hep-ex]].

[40] G. Aad et al. [ATLAS Collaboration], "Search for new phenomena in the dijet mass distribution using $p-p$ collision data at $\sqrt{s}=8 \mathrm{TeV}$ with the ATLAS detector," Phys. Rev. D 91 (2015) 5, 052007 arXiv:1407.1376 [hepex]].

[41] V. Khachatryan et al. [CMS Collaboration], "Search for resonances and quantum black holes using dijet mass spectra in proton-proton collisions at $\sqrt{s}=8 \mathrm{TeV}$," Phys. Rev. D 91 (2015) 5, 052009 arXiv:1501.04198 [hep-ex]].

[42] J. Pumplin, D. R. Stump, J. Huston, H. L. Lai, P. M. Nadolsky and W. K. Tung, "New generation of parton distributions with uncertainties from global QCD analysis," JHEP 0207 (2002) 012 hep-ph/0201195.

[43] R. S. Chivukula, D. A. Dicus and H. J. He, "Unitarity of compactified five-dimensional Yang-Mills theory," Phys. Lett. B 525 (2002) 175 hep-ph/0111016.

[44] R. S. Chivukula and H. J. He, "Unitarity of deconstructed five-dimensional Yang-Mills theory," Phys. Lett. B 532 (2002) 121 hep-ph/0201164.

[45] H. Georgi, "A tool kit for builders of composite models," Nucl. Phys. B266 (1986) 274.

[46] G. Cacciapaglia, C. Csaki, C. Grojean and J. Terning, "Curing the Ills of Higgsless models: The S parameter and unitarity," Phys. Rev. D 71 (2005) 035015 hep-ph/0409126.

[47] D. B. Kaplan, "Flavor at SSC energies: A New mechanism for dynamically generated fermion masses," Nucl. Phys. B 365 (1991) 259.

[48] T. Abe, R. S. Chivukula, E. H. Simmons and M. Tanabashi, "The Flavor Structure of the Three-Site Higgsless Model," Phys. Rev. D 85 (2012) 035015 arXiv:1109.5856 [hep-ph]].

[49] M. E. Peskin and T. Takeuchi, "A New constraint on a strongly interacting Higgs sector," Phys. Rev. Lett. 65 (1990) 964.

[50] M. E. Peskin and T. Takeuchi, "Estimation of oblique electroweak corrections," Phys. Rev. D 46 (1992) 381.

[51] R. S. Chivukula, E. H. Simmons, H. J. He, M. Kurachi and M. Tanabashi, "Ideal fermion delocalization in Higgsless models," Phys. Rev. D 72 (2005) 015008 hep-ph/0504114.

[52] E. Eichten, I. Hinchliffe, K. D. Lane and C. Quigg, "Super Collider Physics," Rev. Mod. Phys. 56 (1984) 579 [Rev. Mod. Phys. 58 (1986) 1065].
[53] K. D. Lane and M. V. Ramana, "Walking technicolor signatures at hadron colliders," Phys. Rev. D 44 (1991) 2678.

[54] E. Eichten and K. D. Lane, "Low - scale technicolor at the Tevatron," Phys. Lett. B 388 (1996) 803 hep-ph/9607213.

[55] K. D. Lane, "Technihadron production and decay in low scale technicolor," Phys. Rev. D 60 (1999) 075007 hep-ph/9903369.

[56] K. Lane and S. Mrenna, "The Collider phenomenology of technihadrons in the technicolor straw man model," Phys. Rev. D 67 (2003) 115011 hep-ph/0210299.

[57] E. Eichten and K. Lane, "Low-scale technicolor at the Tevatron and LHC," Phys. Lett. B 669 (2008) 235 arXiv:0706.2339 [hep-ph]].

[58] H. J. He, Y. P. Kuang, Y. H. Qi, B. Zhang, A. Belyaev, R. S. Chivukula, N. D. Christensen and A. Pukhov et al., "CERN LHC Signatures of New Gauge Bosons in Minimal Higgsless Model," Phys. Rev. D 78 (2008) 031701 arXiv:0708.2588 [hep-ph]].

[59] T. Abe, T. Masubuchi, S. Asai and J. Tanaka, "Drell-Yan Production of Z' in the Three-Site Higgsless Model at the LHC," Phys. Rev. D 84 (2011) 055005 arXiv:1103.3579 [hep-ph]].

[60] C. Du, H. J. He, Y. P. Kuang, B. Zhang, N. D. Christensen, R. S. Chivukula and E. H. Simmons, "Discovering New Gauge Bosons of Electroweak Symmetry Breaking at LHC-8," Phys. Rev. D 86 (2012) 095011 arXiv:1206.6022 [hep-ph]].

[61] R. Casalbuoni, S. De Curtis, D. Dominici, and R. Gatto, "Effective weak interaction theory with possible new vector resonance from a strong higgs sector," Phys. Lett. B155 (1985) 95.

[62] R. Casalbuoni et. al., "Degenerate bess model: The possibility of a low energy strong electroweak sector," Phys. Rev. D53 (1996) 5201-5221, hep-ph/9510431.

[63] K. Lane and A. Martin, "An Effective Lagrangian for Low-Scale Technicolor," Phys. Rev. D 80 (2009) 115001 arXiv:0907.3737 [hep-ph]].

[64] M. Bando, T. Kugo, S. Uehara, K. Yamawaki, and T. Yanagida, "Is rho meson a dynamical gauge boson of hidden local symmetry?," Phys. Rev. Lett. 54 (1985) 1215.

[65] M. Bando, T. Kugo, and K. Yamawaki, "On the vector mesons as dynamical gauge bosons of hidden local symmetries," Nucl. Phys. B259 (1985) 493.

[66] M. Bando, T. Fujiwara, and K. Yamawaki, "Generalized hidden local symmetry and the a1 meson," Prog. Theor. Phys. 79 (1988) 1140.

[67] M. Bando, T. Kugo, and K. Yamawaki, "Nonlinear realization and hidden local symmetries," Phys. Rept. 164 (1988) 217-314.

[68] M. Harada and K. Yamawaki, "Hidden local symmetry at loop: A new perspective of composite gauge boson and chiral phase transition," Phys. Rept. 381 (2003) 1-233, hep-ph/0302103.

[69] R. Barbieri, A. Pomarol, R. Rattazzi and A. Strumia, "Electroweak symmetry breaking after LEP-1 and LEP2," Nucl. Phys. B 703 (2004) 127 hep-ph/0405040.

[70] T. Abe, S. Matsuzaki and M. Tanabashi, "Does the three site Higgsless model survive the electroweak precision tests at loop?," Phys. Rev. D 78 (2008) 055020 arXiv:0807.2298 [hep-ph]].

[71] R. Sekhar Chivukula, N. D. Christensen, B. Coleppa and 
E. H. Simmons, "The Top Triangle Moose: Combining Higgsless and Topcolor Mechanisms for Mass Generation," Phys. Rev. D 80 (2009) 035011 arXiv:0906.5567 [hep-ph]].

[72] T. Abe and R. Kitano, "Phenomenology of Partially Composite Standard Model," Phys. Rev. D 88 (2013) 1, 015019 arXiv:1305.2047 [hep-ph]].

[73] D. K. Hong, S. D. H. Hsu and F. Sannino, "Composite Higgs from higher representations," Phys. Lett. B 597 (2004) 89 hep-ph/0406200].
[74] K. Agashe, R. Contino and A. Pomarol, "The Minimal composite Higgs model," Nucl. Phys. B 719 (2005) 165 hep-ph/0412089.

[75] S. Matsuzaki and K. Yamawaki, "Techni-dilaton at 125 GeV," Phys. Rev. D 85 (2012) 095020 arXiv:1201.4722 [hep-ph]].

[76] K. Lane, "A composite Higgs model with minimal finetuning: The large- $N$ and weak-technicolor limit," Phys. Rev. D 90 (2014) 9, 095025 arXiv:1407.2270 [hep-ph]]. 